\title{
FACETEQ Interface Demo for Emotion Expression in VR
}

\author{
Ifigeneia Mavridou${ }^{1}$, James T. McGhee ${ }^{3}$ Mahyar Hamedi³, Mohsen Fatoorechi ${ }^{3}$, Andrew Cleal ${ }^{3}$, Emili Ballaguer- \\ Balester ${ }^{2}$, Ellen Seiss ${ }^{2}$, Graeme Cox ${ }^{3}$, Charles Nduka ${ }^{3}$ \\ ${ }^{1}$ Centre of Digital Entertainment, Bournemouth University, Bournemouth, UK \\ ${ }^{2}$ Faculty of Science and Technology, Bournemouth University, Bournemouth, UK \\ ${ }^{3}$ 7Emteq Ltd, Sussex Innovation Centre, Science Park Square, Brighton, UK
}

\begin{abstract}
Faceteq prototype v.05 is a wearable technology for measuring facial expressions and biometric responses for experimental studies in Virtual Reality. Developed by Emteq Ltd laboratory, Faceteq can enable new avenues for virtual reality research through combination of high performance patented dry sensor technologies, proprietary algorithms and real-time data acquisition and streaming. Emteq founded the Faceteq project with the aim to provide a human-centered additional tool for emotion expression, affective human-computer interaction and social virtual environments. The proposed demonstration will exhibit the hardware and its functionality by allowing attendees to experience three of the showcasing applications we developed this year.
\end{abstract}

Keywords: virtual reality, facial expression, emotion, EMG, affective computing

Index Terms: H.5.1 [Information Interfaces and Presentation]: Multimedia Information Systems-Artificial, augmented, and virtual realities

\section{INTRODUCTION}

Emotions are principally expressed via facial expressions to exhibit joy, sadness, fear, anger, surprise and disgust. Positive valence is immediately recognisable in a smile, whereas negative valence is indicated by a frown of anger or a scowl of disgust. The scientific study of facial expressions and emotions began with the work of Charles Darwin and Guillaume Duchenne de Bologne in the 19th century. Later, psychologist Paul Ekman pioneered the study of facial expressions of emotions, principally using images. His work led to the categorisation of facial expressions of emotions into discrete archetypes; happiness, sadness, fear, anger surprise and disgust. He co-authored the now famous Facial Action Coding System (FACS) which is now the basis of many emotional research studies, facial animation software packages and emotion analytics businesses.

For facial expression recognition, the most common method is through facial imaging using cameras. However, changes on the position of facial features are the result of muscle contractions, detectable at 1000 times per second from electromyography (EMG) electrodes, in comparison with cameras with sample at 30-60 times per second. EMG allows the detection of baseline muscle tone changes and the monitoring of micro-expressions which may not be observable. Indeed, EMG was used to calibrate camera-based facial expression algorithms [1].
Nowadays, measuring facial expressions and emotional responses using EMG is a fundamental tool for researchers in media, marketing, gaming and psychology. At Emteq, we are studying how emotion recognition technology can be incorporated to Virtual Reality (VR) technology, creating a path to naturalistic social interaction in VR and providing tools for scientific and medical research. At IEEE VR 2017 we will present Faceteq v.05 along with a series of demonstrations that illustrate potential applications in VR.

\section{Demo Description}

Faceteq is a wearable platform that can be adjusted on commercial head-mounted displays (HMDs) (Figure 1). It utilises EMG technology for muscle tone detection to determine facial muscle activations. Unlike standard surface EMG electrodes which require skin preparation, conductive gel and adhesive pads, our prototype consists of dry integrated sensors with 24-bit signal resolution, 1000 samples/sec, no inter-sensor latency and with a signal bandwidth of $20-450 \mathrm{~Hz}$. This version includes integrated 9DOF accelerometer-gyroscope and photo-plethysmograph (PPG) pulse rate sensors and hence provides movement, posture and state analysis, live data streaming to Unity3D via API, real-time signal quality monitoring and supports ASCII data files as well as binary files for post- acquisition data analysis.

Studies within the last decade have demonstrated the benefits of measuring facial EMG, as it can be used 1) as feedback in the interactive design process, 2) to measure involuntary emotional reactions at key product purchase evaluation stages, and 3) to improve user experience during gameplay. We believe our Faceteq platform will be ideally suited for researchers in psychology, human-computer interaction and healthcare, content creators for assessing emotional engagement and developers for gameplay optimization and for the enrichment of interaction-design tools.

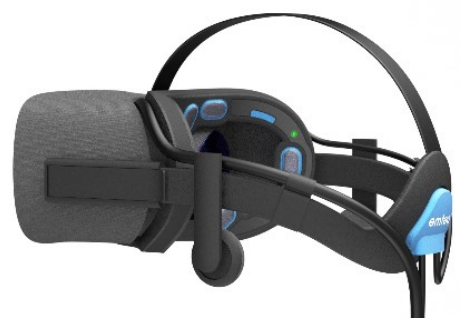

Figure 1: Faceteq v.05 adjusted on an Oculus rift CV1

\subsection{AUdienCE INTERACTION}

In the conference, we plan to show three demonstrations of the use of Faceteq in VR, developed in the Unity3D game engine. Attendees will be asked to wear a commercial HMD and headphones. All navigation and interaction will be accomplished 
though gaze control and facial expressions following a brief calibration.

In the first demo, an avatar will be mirroring the attendee facial expression in real-time (Figure 2). In the second demo, attendees will be able to use facial expressions as an input modality (a model for assistive technology) and react to content, for example a mockup resembling to social media posts, choosing from a list of emoticons by making the according facial expression (Figure 3). The third demo will be a VR cinema, where the attendee will choose from a selection of available videos and watch it in virtual cinema (Figure 4). The system will record the user's reactions to the videos and we will demonstrate our progress in data acquisition for user-experience analysis purposes.

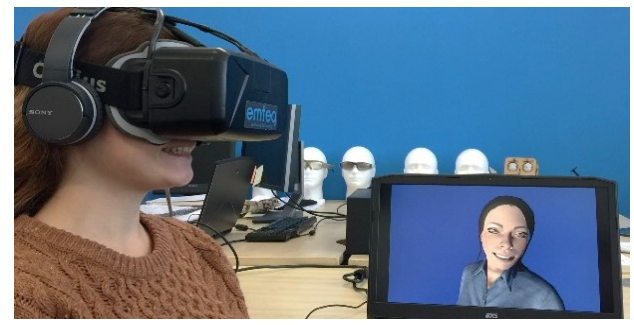

Figure 2: User interacting with Virtual Avatar

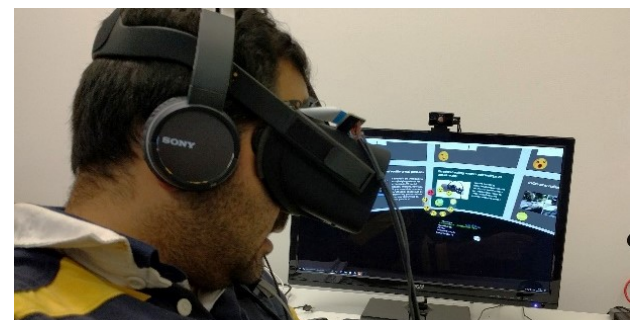

Figure 3: Emoticon selection with facial gestures

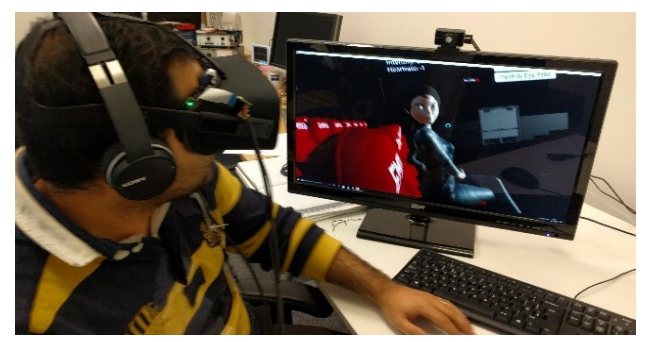

Figure 4: User exploring the VR Cinema demo

\section{FUTURE WORK}

The Faceteq platform and the demonstrations were developed by Emteq Ltd, at Innovation Centre, Sussex University, UK. Emteq's vision is to deliver Virtual Reality that can respond to emotional state, by developing facial wearables that can read and interpret facial gestures and arousal states, using a combination of biometric sensors and artificial intelligence techniques. The company is creating an open platform and is working closely with scientists and researchers from various institutions on numerous projects focused on three principal areas;

a) Emotion Recognition for naturalistic emotion expressioncommunication in social or game environments and for userexperience related content analysis, b) Performance monitoring and drowsiness detection via measurement of emotional state, physiological and behavioural analysis,

c) Medical treatment; by developing technology solutions to enable intervention for healthcare such as in the treatment and rehabilitation of autism spectrum disorder, anxiety, depression, facial paralysis and post-traumatic stress disorder.

Input technologies for VR can be categorised according to the mode of sensing. First generation VR input provides input to the screen via mechanical movement (of the head) to change the point of view. Second generation VR input allows the user to use a limb to influence the VR experience (e.g. pointers, limb trackers). Eye tracking represents the third generation of VR and offers the potential of eye interaction and foveated rendering. Facial or emotional expression enables fourth generation VR and has the potential to greatly improve the user experience.

\section{Conclusion}

Our prototype implementation and the interactive demo concepts will show how facial expressions can be used as an input for interaction in VR as well as for the assessment of user-experience via biometric and behavioural data acquisition. We anticipate that interaction hardware and a new generation of affective avatars will rapidly evolve in the next years.

Faceteq platform and the three demonstrations will be exhibited for the first time at the IEEE VR community where our team will benefit from gathering feedback and learning from the research presented at the conference.

\section{REFERENCES}

[1] J. Cohn and K. Schmidt, The Timing of Facial Motion in Posed and Spontaneous Smiles, International Journal of Wavelets, Multiresolution and Information Processing, 2(2): 121-132, 2004.

[2] Fridlund, A.J. \& Cacioppo, J.T. Guidelines for Human Electromyographic Research. Psychophysiology, 23(5): 567-589, September 1986.

[3] Dimberg, U., Thunberg, M., and Grunedal, S. Facial reactions to emotional stimuli: Automatically controlled emotional responses. Cognition and Emotion, 16(4): 449-471, 2002.

[4] Chanel, G., Rebetez, C., Bétrancourt, M., and Pun, Boredom, engagement and anxiety as indicators for adaptation to difficulty in games. In Proceedings of the 12th international conference on Entertainment and media in the ubiquitous era, pages 13-17. ACM, 2008.

[5] Hazlett, R. L. and Benedek, J. Measuring emotional valence to understand the user's experience of software. International Journal of Human-Computer Studies, 65(4):306-314, April 2007.

[6] Hazlett, R. L. Using Biometric Measurement to Help Develop Emotionally Compelling Games. In Game Usability: Advancing the Player Experience, Morgan. Kaufmann. K. Isbister \& N. Schaffer (Eds.), Burlington, MA, 2008

[7] Larsen, J.T., Norris, C.J., and Cacioppo, J.T. Effects of positive and negative affect on electromyographic activity over zygomaticus major and corrugator supercilii. Psychophysiology, 40(5):776-785, September 2003.

[8] Mandryk, R. L. Physiological Measures for Game Evaluation. In Game Usability: Advancing the Player Experience, Morgan Kaufmann, Isbister \& N. Schaffer (Eds.), Burlington, MA, 2008.

[9] Mandryk, R. L. and Atkins, M. S. A fuzzy physiological approach for continuously modeling emotion during interaction with play technologies. International Journal of Human-Computer Studies. 65(4): 329-347, April 2007. 